MINI-SYMPOSIUM

\title{
Endothelial dysfunction and cardiovascular disease: the role of predictive adaptive responses
}

\author{
$M$ Hanson, $P$ Gluckman
}

Heart 2005;91:864-866. doi: 10.1136/hrt.2004.047381

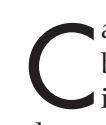
ardiovascular disease remains a major cause of morbidity and mortality in developed societies. It is also an increasing problem in developing societies, especially those in which transitions are occurring-for example, the migration of young people from rural to urban environments or the increasing availability of fast food of high calorific content. It is clear that such rapid changes in the incidence of cardiovascular disease, in some instances within a generation, cannot be explained in purely genetic terms. This does not deny the role of genetic factors (for example, single nucleotide polymorphisms) which convey susceptibility to disease, but emphasises that additional environmental factors must also play a major role. This idea is of course not new, although for many years such environmental contributions to disease risk have been considered to be largely adult "lifestyle" factors such as smoking, diet, and physical activity level. However, the results of interventions aimed at reducing such risk factors in adults have so far been disappointing. Pharmacological interventions such as statins have been shown to confer benefit in reducing occurrence of cardiovascular episodes in those at high risk (for example, type 2 diabetes or after a previous myocardial infarction ${ }^{1}$ ) but their use more widely is debated..$^{2}$ The question therefore arises of how to target such preventative measures or interventions in otherwise healthy individuals (or sections of the population) who are at high risk. Such considerations must take into account the full range of risk factors: the series of short papers in this symposium focus on the risks associated with the early environment, prenatally and in childhood.

The contribution of the environment to risk of disease has been widely discussed, ${ }^{3}$ particular emphasis usually being placed on the association between low birth weight and components of the metabolic syndrome such as hypertension or type 2 diabetes. The strength of the association has been challenged largely on the basis of the size of the correlation between birthweight and adult blood pressure. ${ }^{4}$ We believe that such debate is sterile as it centres around the use of surrogate measures, both of prenatal environment (birthweight) and later cardiovascular disease (blood pressure). When disease is the outcome measure, the outcome is clear. ${ }^{5}$ A wide range of mechanistic investigations in animals has shown that adult metabolic, endocrine, and cardiovascular function can be "programmed" by the prenatal environment in the absence of a change in birthweight, and of course blood pressure in middle age may not be significantly elevated even in those who will subsequently have cardiovascular incidents-for example, their renal capacity may be impaired but they may currently be able to compensate. It is therefore clear that aspects of the prenatal environment can programme risk of disease in later life without necessarily being manifest in middle age. This insight needs to be explored further if its implications for prevention of disease are to be exploited. The range of research currently being undertaken around the world into the developmental origins of disease can be seen from the papers presented at the Second World Congress on Fetal Origins of Adult Disease held in June 2003. ${ }^{67}$

\section{THE HEART AND ITS ENDOTHELIUM}

The papers in this symposium are concerned with early effects on cardiac development, in particular growth and the development of the coronary circulation and the role of the vascular endothelium. Aside from its obvious role in cardiovascular disease, the heart is one of the few organs in the body in which the complement of cells (cardiomyocytes) is essentially formed before birth. There may be progenitor stem cells retained ${ }^{8}$ into later life but they cannot usually repair the damage of an infarct, for example. In addition, once the period of cardiac myogenesis is complete, the heart must respond to increased load by hypertrophy rather than hyperplasia. The growth, and hence later function of capacity, of the heart depends on its blood supply. In this regard the endothelial cells are particularly important. ${ }^{9}$ A range of animal models has demonstrated changes in vascular endothelial cell function, and associated vascular smooth muscle responses, in peripheral vessels from a range of tissues. Particular emphasis has also been placed on changes in low mediated vasodilatation in children and young adults. ${ }^{10-12}$ Vascular endothelial cells play an important role in normal physiological responses in respect of:

- vessel calibre

- vascular growth and remodelling, including stem cell activation

- tissue and organ growth

- tissue metabolism

- immune responses

- blood fluidity

- platelet and white cell stickiness

- vascular permeability.

Moreover, vascular endothelial cell dysfunction is known to play a role in a range of disease states including:

- hypertension

- atherogenesis

- type 2 diabetes

- pre-eclampsia

- coronary heart disease

- metabolic syndrome-obesity.

It is therefore possible to argue that changes in vascular endothelial cell function precede, accompany, or follow changes in cardiac development and function. As a general rule disease results when an individual's capacity to mount an adequate homeostatic response to a challenge is exceeded. Thus either changes in cardiac growth/function and/or 


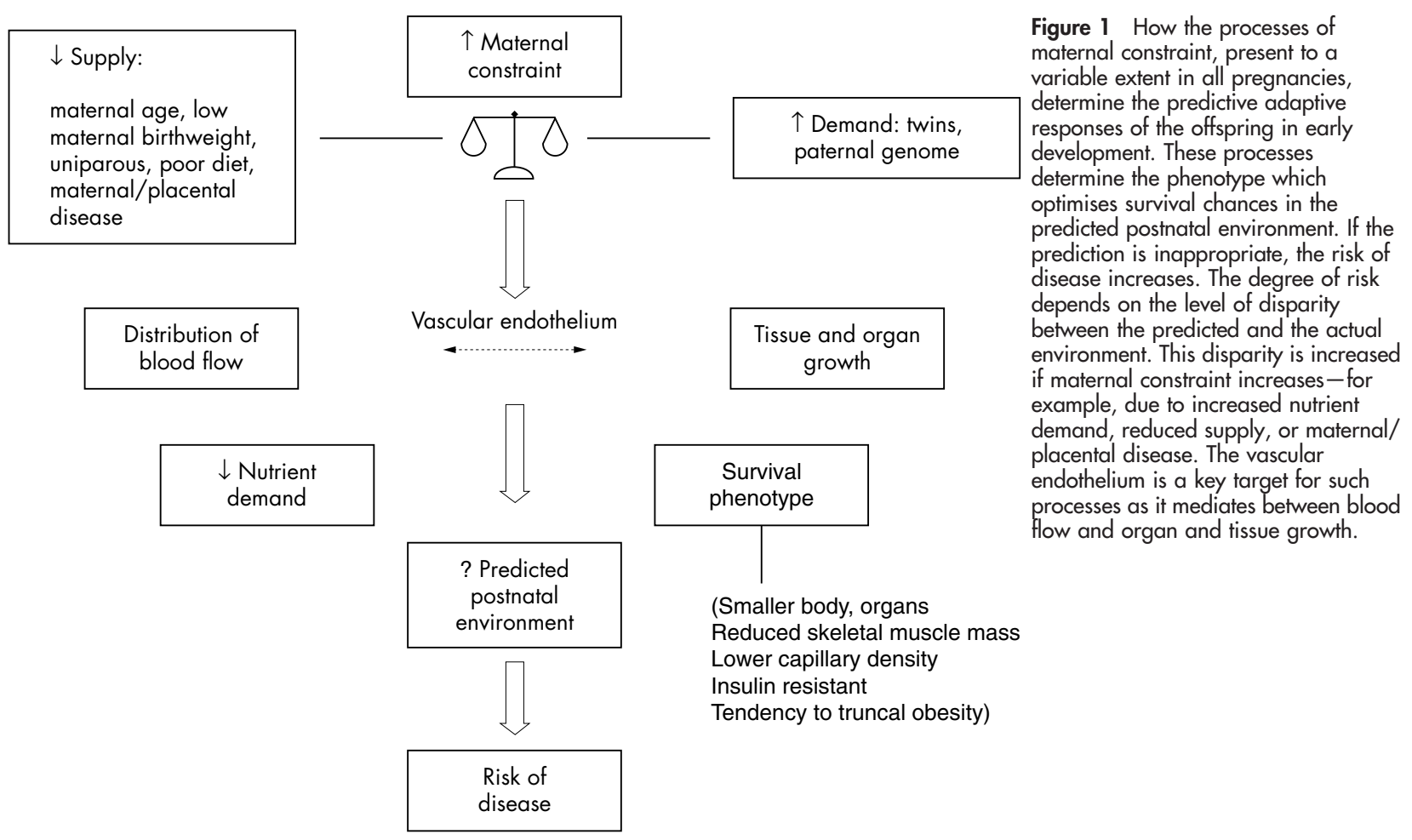

endothelial function may determine the risk of disease. This may be incorporated into a general model (fig 1).

\section{THE SURVIVAL PHENOTYPE}

In the absence of clear "lifestyle" influences, the different prevalence of diseases such as type 2 diabetes in populations have been ascribed to other factors. Several of them involve an evolutionary perspective. One influential concept was that of the thrifty genotype. ${ }^{13}$ Here evolution is proposed to have selected for genes (for example, those associated with insulin resistance) which confer an advantage in a deprived nutritional environment. Such thrifty genes would be selected because they would promote chances of survival to reproductive age. Evolutionary selection (whether by natural or sexual selection) can only work on the phenotypic range of individuals of the species, up until the time when their reproductive capacity ceases. It therefore indirectly selects for genotype which gives a phenotypic advantage in terms of survival to reproduce in an environment. Because it is now clear that a plethora of factors intercede between genotype and phenotypic expression, and because evolutionary processes must operate on the phenotype rather than the genotype, it is more appropriate to consider a thrifty phenotype model. This was advanced by Hales and Barker. ${ }^{14}$ In such a model diseases will result from the operation of two additional processes. The first occurs when the environment of the thrifty phenotype offspring becomes rich. Hence a fast food culture in which the diet is unbalanced, high in calories and fat, and requires little energy expenditure to obtain, will produce disease. The second results from the increasing longevity of humans beyond their reproductive age. Diseases therefore result from pathophysiological processes which evolution, by definition, has not been able to select against. This principle is really an extension of the biological trade-off theory of Williams ${ }^{15}$ as expanded by Kirkwood. ${ }^{16}$ We have recently incorporated these concepts into a more general theory - that of predictive adaptive responses. ${ }^{17}$ It builds on the thrifty phenotype model by emphasising the processes by which the mother restricts fetal growth, even in normal uncomplicated pregnancies. This process is termed maternal constraint. ${ }^{18}$

\section{THE IMPORTANCE OF THE TRANSITION}

The most important aspect of the predictive adaptive responses model is that the risk of disease depends on the degree of mismatch between the predicted postnatal environment and that which actually exists. The prediction is always slightly incorrect because evolution has selected processes which programme the fetus towards the survival phenotype; in other words, the default position is to develop a phenotype which anticipates a slightly worse postnatal environment then actually exists. This failsafe strategy is produced by the processes of maternal constraint, which evolved to reduce the risk of obstructed labour and maternal death intrapartum, but which have been preserved because of their postnatal survival to hominids who were exposed to uncertain nutrition during their evolution as hunter-gatherers. ${ }^{17}$ Physiological situations which increase the degree of maternal constraint therefore increase the mismatch and thus the likelihood that an inappropriate predictive adaptive response leads to disease. Such situations include those in which fetal demand is increased relative to maternal/ placental supply-for example, twins or a male fetus from a large father, and those in which maternal supply is reduced in relation to fetal demand-primiparous pregnancy (especially older women), teenage pregnancy, low maternal size/ body weight, and unbalanced maternal diet or body composition. Disease states in pregnancy will produce equivalent effects such as pre-eclampsia or pregnancy induced hypertension or placental disease, which reduces supply, and gestational diabetes mellitus, which increases it. The latter is particularly interesting as normal pregnancy is a mildly diabetogenic state, due to the action of hormones such as placental lactogen. Moreover gestational diabetes mellitus (GDM) is more likely in women who themselves were low birthweight. ${ }^{19}$ 
The pre- and postnatal mismatch, and thus the risk of disease associated with an inappropriate predictive adaptive response, is also increased when the postnatal environment has changed. This is particularly the case with the current level of high calorie/fat fast-food consumption combined with the low levels of physical energy expenditure needed to acquire it. Such rapid transitions in nutrition are manifest both in developing and developed societies. The concomitant increase in the level of obesity in youngsters and the epidemic of cardiovascular disease and type 2 diabetes in, for example, the Indian subcontinent are well known. The effects of the transition are extremely worrying; while the incidence of coronary heart disease has been falling steadily in western societies over the last few decades we believe that it may now not fall further and in fact is likely to increase again as a result of the speed of environmental transition.

\section{THE FUTURE}

While the epidemic of disease associated with the metabolic syndrome is daunting, the rapid pace of research into the mechanisms by which predictive adaptive responses contribute to the risk of such disease offers hope of intervention. It will be essential to identify phenotypic characteristics, in individuals or sections of the population, which confer greater risk. A major need here will be to establish intermediate outcome measures which can be used reliably in children or adolescents, for example. Such measures will also be essential for evaluating the efficacy of interventions. We believe that measures of endothelial function will play a major role here. It may be that the therapeutic approaches being examined for the use of stem cells for vascular endothelial cell regeneration ${ }^{20}$ or gene therapy to promote angiogenesis $^{21}$ will give insights which can be applied to correct endothelial dysfunction arising from inappropriate PARs. In the meantime the emphasis must be on understanding the mechanisms by which endothelial function is changed and on considering possible interventions.

\section{Authors' affiliations \\ M Hanson, Centre for Developmental Origins of Health and Disease, University of Southampton, Princess Anne Hospital, Southampton, UK P Gluckman, Liggins Institute, Auckland, New Zealand}

Correspondence to: Professor Mark Hanson, Centre for Developmental Origins of Health and Disease, University of Southampton, Level F
(MP887), Princess Anne Hospital, Coxford Road, Southampton, S016 5YA, UK; m.hanson@soton.ac.uk

\section{REFERENCES}

1 Medical Research Council, British Heart Foundation. MRC/BHF heart protection study. Lancet 2003;361:2005-16.

2 Wald NJ, Law MR. A strategy to reduce cardiovascular disease by more than $80 \%$. BMJ 2003;326:1419. See comment in BMJ 2003;326:1407-8; see also BMJ 2003;327:807-8; discussion 809; author reply 809-10.

3 Barker DJ. Fetal programming of coronary heart disease. Trends Endocrinol Metab 2002; 13:364-8.

4 Huxley R, Neil A, Collins R. Unravelling the fetal origins hypothesis: is there really an inverse association between birthweight and subsequent blood pressure? Lancet 2002;360:659-65.

5 Curhan GC, Willett WC, Rimm EB, et al. Birth weight and adult hypertension, diabetes mellitus, and obesity in US men. Circulation 1996;94:3246-50.

6 Anon. Second World Congress of Fetal Origins of Adult Disease. Ped Res 53(6)(suppl) [abstracts].

7 Hanson M, Gluckman P, Bier D, et al. Report on 2nd World Congress on Fetal Origins of Adult Disease, Brighton UK, 7-10 June 2003. Ped Res 2004;56:311-17.

8 Mery A, Papadimou E, Zeineddine D, et al. Commitment of embryonic stem cells toward a cardiac lineage: molecular mechanisms and evidence for a promising therapeutic approach for heart failure. J Muscle Res Cell Motil 2003;24:269-74.

9 Brutsaert DL. Cardiac endothelial-myocardial signalling: its role in cardiac growth, contractile performance, and rhythmicity. Physiol Rev 2002;83:59-115.

10 Martin H, Hu J, Gennser G, et al. Impaired endothelial function and increased carotid stiffness in 9-year-old children with low birthweight. Circ 2000; 102:2739-44.

11 Martin H, Gazelius B, Norman M. Impaired acetylcholine-induced vascular relaxation in low birth weight infants: implications for adult hypertension? Pediatr Res 2000;47:457-62.

12 Leeson CP, Kattenhorn M, Morley R, et al. Impact of low birth weight and cardiovascular risk factors on endothelial function in early adult life. Circulation 2001;103:1264-8.

13 Neel JV. Diabetes mellitus: a "thrifty" genotype rendered detrimental by "progress"? Am J Hum Genet 1962;14:353-62.

14 Hales CN, Barker DJ. The thrifty phenotype hypothesis. Br Med Bull 2001;60:5-20.

15 Williams GC. Pleiotropy, natural selection, and the evolution of senescence. Evolution 1957;11:398-411.

16 Kirkwood TBL. Evolution of ageing. Nature 1977;270:301-4.

17 Gluckman PD, Hanson MA. The fetal matrix-evolution, development and disease. Cambridge: Cambridge University Press, 2004.

18 Gluckman PD, Morel PCH, Ambler GR, et al. Elevating maternal insulin-like growth factor-l in mice and rats alters the pattern of fetal growth by removing maternal constraint. J Endocrinol 1992;134:R1-3.

19 Innes KE, Byers TE, Marshall JA, et al. Association of a woman's own birth weight with subsequent risk for gestational diabetes. JAMA 2002;287:2534-41.

20 Isner JM, Asahara T. Angiogenesis and vasculogenesis as therapeutic strategies for postnatal neovascularization. J Clin Invest 1999;103:1231-6.

21 Dzau VJ. Predicting the future of human gene therapy for cardiovascular diseases: what will the management of coronary artery disease be like in 2005 and 2010? Am J Cardiol 2003;92(9B):32N-5N.

\section{FROM BMJ JOURNALS}

\section{Coxsackievirus B3 sequences in the myocardium of fatal cases in a cluster of acute myocarditis in Greece}

\section{N Spanakis, E N Manolis, A Tsakris, S Tsiodras, T Panagiotopoulos, G Saroglou, N J Legakis}

Please visit the Heart website [www.heartinl. com] for a link to the full text of this article.
Aim: The investigation of three fatal cases during a nationwide cluster of cases of an upper respiratory tract infection (URTI) associated with myocarditis and/or pericarditis in Greece in 2002.

Methods: In the three women who died, necropsies were performed and tissue sections were taken for histological examination, antigen detection by immunohistochemistry and indirect immunofluorescence assay (IFA), amplification of viral genomes by nested reverse transcription polymerase chain reaction (RT-PCR), and sequence analysis.

Results: All samples showed histological signs of active myocarditis. Immunohistochemistry revealed the presence of the enterovirus VPl family of proteins and IFA revealed the presence of coxsackievirus B3 antigen. Nested RT-PCR amplified enteroviral alleles of the 5'untranslated region which were identical to each other and to the coxsackievirus B3 sequences.

Conclusions: This study provides pathological evidence of enteroviral infection among fatal myocarditis cases in a nationwide URTI cluster of cases associated with myocarditis and/or pericarditis.

A Journal of Clinical Pathology 2005;58:357-360. 